\title{
MULTIPLE AND SINGLE GREEN AREA MEASUREMENTS AND CLASSIFICATION USING PHANTOM IMAGES IN COMPARISON WITH DERIVED EXPERIMENTAL LAW
}

\author{
Naser A. M. Abu-Zaid \\ Electrical and Communication Engineering Departments, An-najah National University, Nablus, Palestinian territories \\ naserzaid@najah.edu \\ naser_res@yahoo.com
}

KEY WOORDS: Adaptive weighted distances algorithm, Object classification, Curve fitting, Arial images.

\begin{abstract}
:
In many circumstances, it is difficult for humans to reach some areas, due to its topography, personal safety, or security regulations in the country. Governments and persons need to calculate those areas and classify the green parts for reclamation to benefit from it.To solve this problem, this research proposes to use a phantom air plane to capture a digital image for the targeted area, then use a segmentation algorithm to separate the green space and calculate it's area. It was necessary to deal with two problems. The first is the variable elevation at which an image was taken, which leads to a change in the physical area of each pixel. To overcome this problem a fourth degree polynomial was fit to some experimental data. The second problem was the existence of different unconnected pieces of green areas in a single image, but we might be interested only in one of them. To solve this problem, the probability of classifying the targeted area as green was increased, while the probability of other untargeted sections was decreased by the inclusion of parts of it as non-green. A practical law was also devised to measure the target area in the digital image for comparison purposes with practical measurements and the polynomial fit.
\end{abstract}

\section{INTRODUCTION}

There are many reasons to measure and classify arbitrarily shaped pieces of land including trade, agriculture, division among partners, security or reclamation (Liu et al., 2017; Lopes et al., 2017; Santos et al., 2016; Svatonova and Kolejka, 2017). Measuring regular spaces is often a simple process because the land takes a regular or semi-regular shape. But, measuring irregular shapes of land is not as easy. Of course, it's possible to resort to a surveyor, but it is either expensive or unfeasible to reach the targeted land due to security or safety reasons. The methods of measuring spaces vary from simple ones based on math basics to complex ones. The simple methods includes division of the irregular land into regular ones then summing there areas, or to use aplanimeterwhich requires a precise map, or to use measuring wheel which involves work in the field, or using transparent paper. More complicated methods use GPS or satellite photos (Lopes et al., 2017) (Google earth) with appropriate software (Gonzalez and Woods, 2008; Protiere and Sapiro, 2007).

Most of these methods lack the abilityto classify green land or calculate small areas of low resolution or they are not automated. For these reasons, the use of low-altitude aerial images (Liu et al., 2017; Svatonova and Kolejka, 2017) to classify and calculate green spaces will be discussed in this paper. Here, two things will be done. The first, derive a practical law to calculate the area from the images taken by phantom plane (Audebert et al., 2017) at different altitudes. The second, adapting the measurements at variable altitudes to a polynomial ("Mathworks Corporations," 2017), classification of part(s) of an image as green land(s), and calculate the area automatically.

\section{METHODOLOGY, MOTIVATION, ALGORITHMS AND RESULTS}

\subsection{Practical law for variable altitudes}

In this paragraph, a practical law has been derived based on the completed measurements of the phantom for the municipal football stadium shown in Figure 1. The municipal stadium has been chosen because of its standard lengths which reduce the error rate in the law as well as the ease of comparison with real lengths. Table 1 was obtained after taking a series of aerial photographs of the stadium at variable heights. Based on those measurements, the practical formula in equation (1) was derived to calculate the physical length in meters. The denominator in the formula is a fixed scaling factor. Despite the small measurement error in the number of pixels, the law gives accurate results to calculate area at variable altitudes, as will be shown in later sections.

$$
l=\frac{l_{p} h}{2215.3846154}
$$

Where $\quad l$ : Actual length of a particular section. $l_{p}$ : Length in pixels of that section. $h$ : Actual height of phantom when image is taken. 


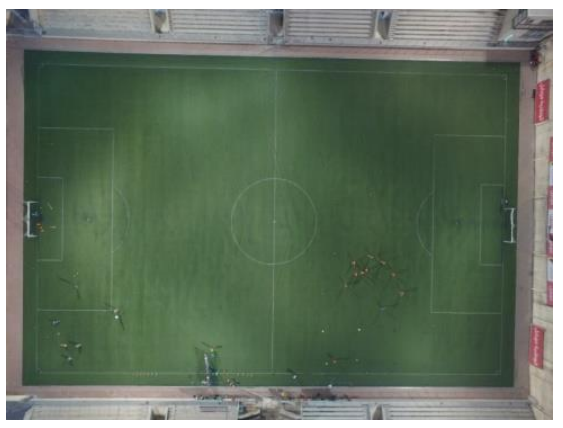

Figure 1. Municipal football stadium used for deriving the practical law

\subsection{Polynomial fit to measurements}

To program and automate the whole process, a set of images was taken at variable altitudes as shown in Table 2 . In this table, the number of pixels per unit length is included. This value is the base stone in calculating the area according to equation (2). Because the number of pixels varies with distance from lens, a $4^{\text {th }}$ degree polynomial was fitted to available measurements as shown in equation (3). To examine the validity of the coupling of polynomial with measurements and the amount of associated error, the results from pairing polynomial was compared with measurements taken for a given region, and results are shown in Figure 2. Clearly the error can be reduced by increasing the polynomial degree and/or increasing the accuracy of the measurements, which is not the subject of this research.

$$
S=\frac{n_{p}}{n_{m}^{2}}
$$

Where $S$ : Physical area in squared meter.

$n_{p}$ : Number of pixels in an image or part of an image.

$n_{m}$ : Number of pixels per unit length.

\begin{tabular}{c|c}
\hline Number in Pixels & Height in meters \\
\hline 3920 & 11 \\
\hline 2808 & 15 \\
\hline 2138 & 20 \\
\hline 1484 & 30 \\
\hline 1094 & 40 \\
\hline 879 & 50 \\
\hline 623 & 70 \\
\hline
\end{tabular}

Table 1. Data used for experimental law derivation

\begin{tabular}{c|c|c|c|l}
\hline Image & $l_{p}$ & $h$ & \multicolumn{1}{|c|}{$n_{m}$} & $l$ (Exp. law) \\
\hline IMAGE 1 & 3217 & 15 & 164.974359 & 21.78178 \\
\hline IMAGE 2 & 1733 & 25 & 88.87179487 & 19.55643 \\
\hline IMAGE 3 & 1257 & 35 & 64.46153846 & 19.85886 \\
\hline IMAGE 4 & 989 & 45 & 50.71794872 & 20.08907 \\
\hline IMAGE 5 & 813 & 55 & 41.69230769 & 20.18386 \\
\hline IMAGE 6 & 685 & 65 & 35.12820513 & 20.0981 \\
\hline
\end{tabular}

Table 2. Measurements used for polynomial derivation and comparison with experimental law

$$
\begin{gathered}
n_{m}(h)=5.433 \times 10^{-5} h^{4}-0.011 h^{3}+0.8258 h^{2}- \\
28.29 h+43.8
\end{gathered}
$$

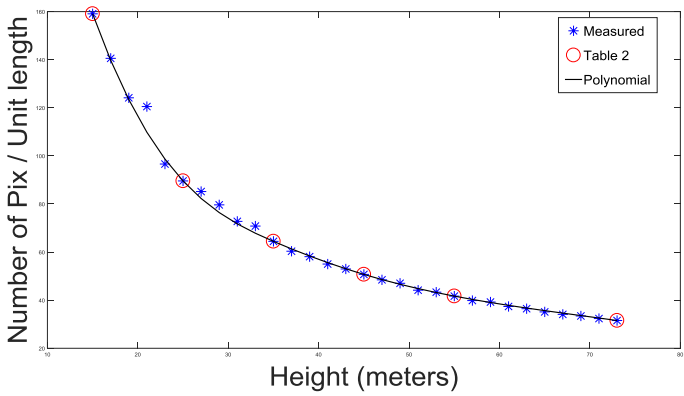

Figure 2. Comparison of Polynomial fitting with measurements

\subsection{Green area classification and measurements}

To calculate the green part(s) of a land in an image requires segmenting and separating it from the rest of the image. Therefore, a segmentation algorithm known as adaptive weighted distance algorithm was used for its accuracy, robustness, and ease of use (Gonzalez and Woods, 2008; Lopes et al., 2017). Most of the material in the following derivation is borrowed from (Protiere and Sapiro, 2007). Let $\left\{l_{1}, \ldots \ldots, l_{N l}\right\}$ be a set of labels of an image $\Omega$. Assuming each label contains several components, and letting $l_{i}^{j}$ be the $j^{\text {th }}$ component the label $l_{i}$. Then the weight function for this component is given by:

$$
W_{i}^{j}=\sum_{k \neq i} \sum_{l} W_{l_{i}^{j} \mid l_{k}^{l}}
$$

Where $W_{l_{i}^{j} \mid l_{k}^{i}}$ is the weight function for component label $l_{i}^{j}$ when competing only with $l_{k}^{l}$. The weighted geodesic distance between two pixels $s$ and $t$ of the image $\Omega$ over the path $C_{s, t}$ connecting both pixels is defined by:

$$
d(s, t):=\underbrace{\min }_{C_{s, t}} \int_{s}^{t}\left|W_{i}^{j}(x) \cdot \dot{C}_{s, t}(x)\right| d x
$$

If $G_{i}$ is the response of the $i^{\text {th }}$ filter applied to the image, then the probability for a pixel $x$ to be assigned to label $l_{1}$ based on the $i^{\text {th }}$ channel filter is given by:

$$
P_{1 \mid 2}:=\operatorname{Pr}\left(x \in l_{1}\right)=\sum_{i=1}^{N_{c}} W_{1} P_{1 \mid 2}^{i}(x)
$$

Where $N_{c}$ is the number of channel filters and $P_{1 \mid 2}^{i}$ is given by:

$$
P_{1 \mid 2}^{i}(x)=\frac{p_{1}^{i}\left(F_{i}(x)\right)}{p_{1}^{i}\left(F_{i}(x)\right)+p_{2}^{i}\left(F_{i}(x)\right)}
$$

Where $p_{j}^{i}$ is the probability density function (PDF) on channel filter $F_{i}$. This process could be repeated for all labels.

The image in Figure 3 shows several separate green zones. In that image, red and blue rectangles, which indicate the primitive seeds required by the algorithm, are also shown. Red 
rectangles point to foreground scribbles of image, while blue scribbles point to background scribbles of image. With a quick look at Figure 4, it is clear to the reader the precise separation of blue-colored cultivated areas. In addition, the program works to calculate this green space and gives approximately $1469.8995 \mathrm{~m}^{2}$.

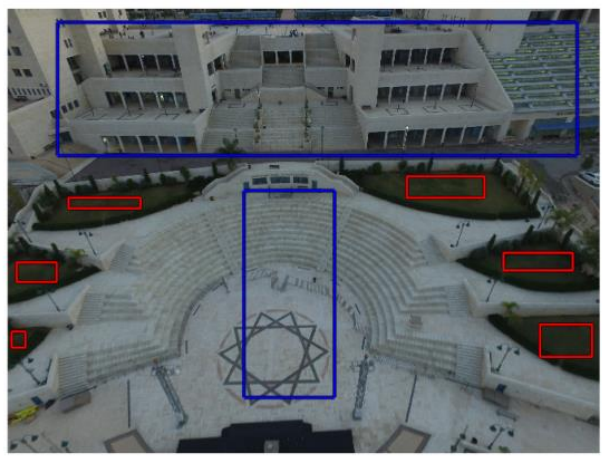

Figure 3. University open theater image with initial seed rectanglesused to apply segmentation and space calculation algorithm of multiple green areas

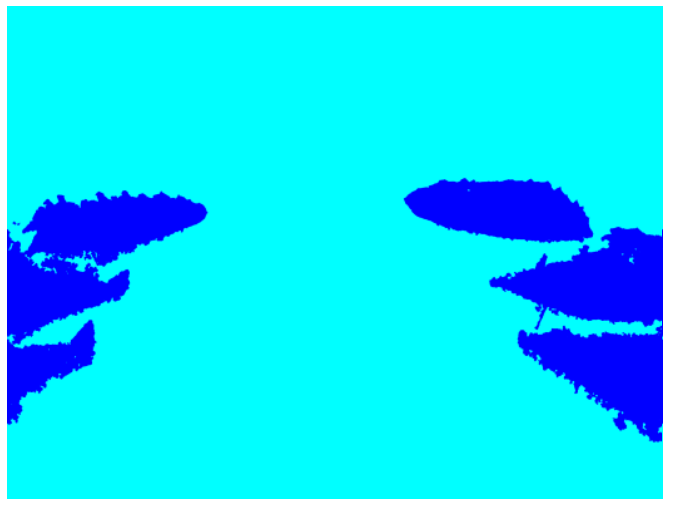

Figure 4. Segmented image with multiple cultivated areas shown in blue

Sometimes, it's needed to classify and calculate the area of one piece of green land only within a set of pieces. To separate this piece from others, the probability of classification as a green was increased by choosing the largest possible part of it within the foreground scribbles. For other areas, the probability of being green was reduced by choosing small parts of them within the background scribbles. Figure 5 illustrates these scribbles, and Figure 6 shows results of classification. Note how the seeds of background purposely includes parts of green zones which are not part of the targeted area, while only one single green area is seeded. The area of this green piece of land was calculated by program to be approximately $219.9465 \mathrm{~m}^{2}$. It should be noted that areas of multiple regions could be calculated in two ways. First, Split the image into different areas, then classify and calculate the spaces through implantation of the program once as done in previous illustration. Second: divide the image into several images, and then classify and calculate the space for each section separately, this requires the repeated implementation of the program, but it increases the accuracy of the calculations.

\subsection{Algorithm}

An overview of the operational strategy adapted for the automated process is shown in Figure 7. The preprocessing part is straight forward using Matlab curve fitting functions (Gonzalez and Woods, 2008). A spline or a rational could be implemented to increase the accuracy of the proposed method. Mobile device connected to a computer, can be used to retrieve data from phantom on line and feed it to the software. May be the difficult job is the choice of a classifier from the plenty of available ones that is most suitable for use over small areas, or to combine several classifiers to improve the accuracy.

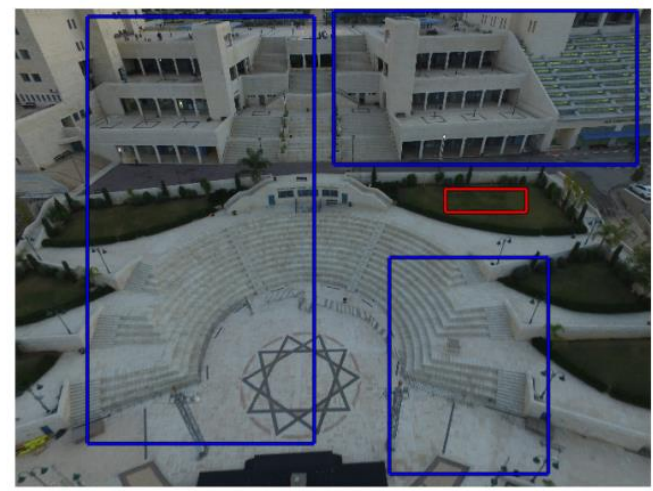

Figure 5. University open theater image with initial seed rectangles used to apply segmentation and space calculation algorithm of single green area

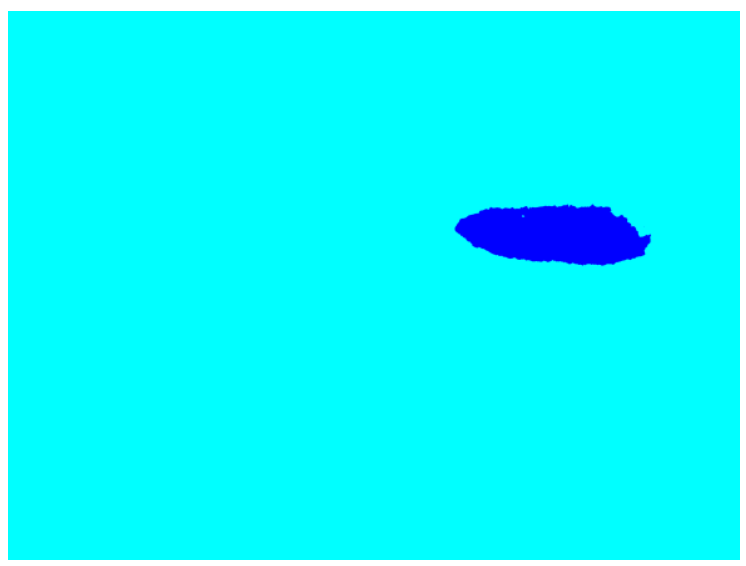

Figure 6. Segmented image with single cultivated area shown in blue

\section{CONCLUSION}

In this applied research, a fully automated method was developed to classify and calculate the fertile land areas using digital images taken from a low-altitude phantom aircraft. In 
addition to deriving a practical law, there has also been a polynomial fitting to available data. The results were concise, and the algorithm was fast and completely automated. It should be noted that this method can be used to classify and calculate the flat area of any object(s) in the image. The algorithm is not limited to farmland, it can be used for any object as long as they can be classified by the algorithm. Anyone can do these classifications and calculations while he is lying at home without resorting to any experts or paying any costs. All it needs is a small plane equipped with a digital camera and
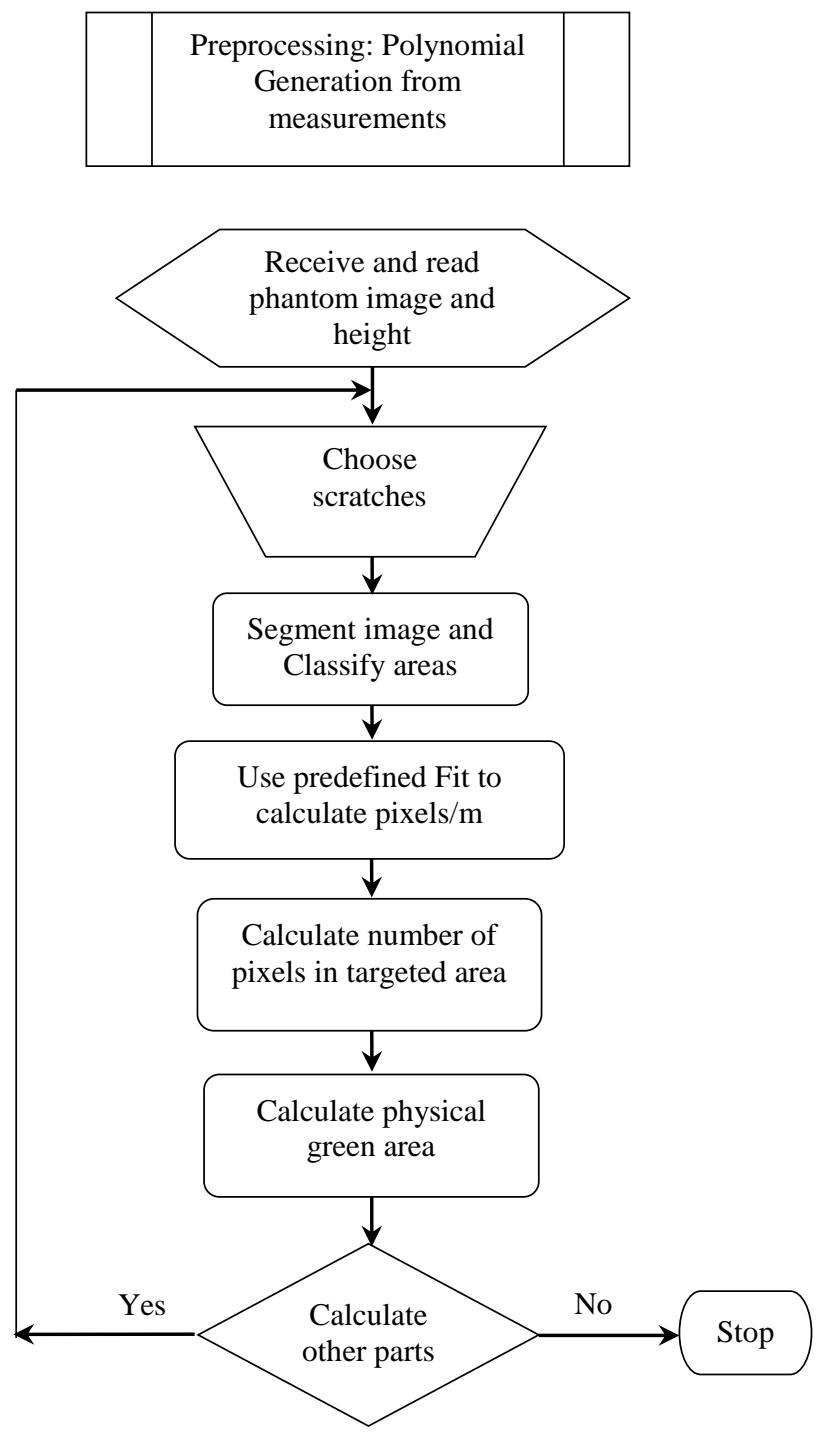

Figure 7. Image classification and area calculation algorithm

\section{AKNOWLEDMENT}

I would like to thank my students Eng. Mohammad Askalan and Eng. Ali Aslan for providing me with the data set needed for this applied research.

\section{REFERENCES}

Audebert, N., Le Saux, B., Lefèvre, S., 2017. Segment-beforeDetect: Vehicle Detection and Classification through Semantic Segmentation of Aerial Images. Remote Sens. doi:10.3390/rs9040368

Gonzalez, R.C., Woods, R.E., 2008. Digital Image Processing.Pearson Inc.

Liu, Y., Nguyen, D.M., Deligiannis, N., Ding, W., Munteanu, A., 2017. Hourglass-ShapeNetwork Based Semantic Segmentation for High Resolution Aerial Imagery. Remote Sens. 1-24. doi:10.3390/rs9060522

Lopes, M., Fauvel, M., Girard, S., Sheeren, D., 2017. Objectbased classification of grasslands from high resolution satellite image time series using Gaussian mean map kernels To cite this version: Object-based classification of grasslands from high resolution satellite image time series using Gaussian mea. Remote Sens. 1-24.

Mathworks Corporations, $2017 . \quad$ URL http://www.mathworks.com

Protiere, A., Sapiro, G., 2007. Interactive Image Segmentation via Adaptive Weighted Distances, in: IEEE TRANSACTIONS ON IMAGE PROCESSING. pp. 1046-1057. doi:10.1109/TIP.2007.891796

Santos, T., Tenedório, J.A., Gonçalves, J.A., 2016. Quantifying the City' s Green Area Potential Gain Using Remote Sensing Data. Sustainability 8, 1-16. doi:10.3390/su8121247

Svatonova, H., Kolejka, J., 2017. Comparative Research of Visual Interpretation of Aerial Images and Topographic Maps for Unskilled Users : Searching for Objects Important for Decision-Making in Crisis Situations. ISPRS Int. J. GeoInformation. doi:10.3390/ijgi6080231

distance sensor, with images fed into a computer program. Google earth images are not appropriate for such processing, since small area classifications and calculations requires high resolution images at low altitudes. 